IEEE Transactions on Nuclear Science, Vol. NS-28, No. 1, February 1981

\title{
A GAS IONIZATION SAMPLING ELECTROMAGNETIC SHOWER DETECTOR
}

M. Atac, D. Hanssen, H. Jensen, A. Tollestrup, and J. Urish

Fermi National Accelerator Laboratory, Batavia, IL 60510*

R. G. Kennett and R. Walker

California Institute of Technology, Pasadena, CA 91125

L. Nodulman

Argonne National Laboratory, Argonne, II 60439

\section{Abstract}

The results of the tests of a prototype ionization sampling calorimeter are presented. Using proportional chambers interleaved with lead and sampling every 0.68 radiation length, we found an energy resolution of $24 \% / \sqrt{E}$. Measurements of position resolution, lateral shower development, and hadron rejection are discussed.

\section{Introduction}

The tests of a proportional wire chamber calorimeter were undertaken as part of the prototyping work for the Colliding Beam Detector project at Fermilab. Ease of construction, spatial resolution, and the ease with which towers may be constructed from cathode pads make this an attractiye system for our end cap calorimetry. Previous work ${ }^{1}, 2$ has shown the practicality of this technique in the construction of $4 \pi$ detectors.

\section{Calorimeter Construction}

The calorimeter was built from 34 proportional wire chambers interleaved with lead plates. The chambers, see Fig. 1, had a $12 \mathrm{~mm}$ gap with 50 micron gold plated tungsten sense wires placed every $6 \mathrm{~mm}$. The cells were separated by 100 micron opper-beryllium field wires at ground potential. Cathode strips run orthogonally to the wires on one side of the chambers. They were $6 \mathrm{~mm}$ center to center with a $1 \mathrm{~mm}$ gap between strips. The chambers were filled with a 508-50\% mixture of argon and ethane for all studies.

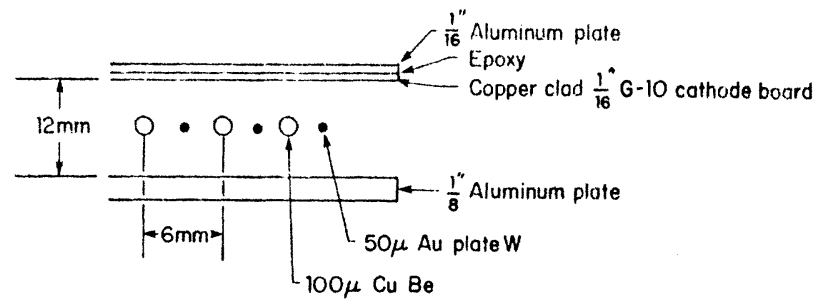

Fig. 1. Chamber construction.

All chambers were tested prior to use. Gain measurements along wires and from wire to wire within a given chamber showed an r.m.s. variation of less than $2.5 \%$ over an active area greater than $20 \times 20 \mathrm{~cm}^{2}$. overall chamber gain was measured by averaging the response of the central wires in each chamber. On the basis of those measurements we were able to segregate the chambers into two groups of 8 and one group of 18 with a total gain variation within each group of not greater than $6 \%$. When the calorimeter was assembled, we alternated "high gain" and "low gain" chambers within each group.

Measurements of gas gain as a function of several parameters resulted in the empirical relationship:

$$
\frac{\Delta G}{G}=15.8 \frac{\Delta E}{E}-15 \frac{\Delta P}{P}+6.3 \frac{\Delta T}{T}-1.3 \frac{\Delta t}{t}
$$

Where $\mathrm{G}$ is gas gain, $\mathrm{E}$ is high voltage, $\mathrm{P}$ is gas pressure, $T$ is temperature, and $t$ is gap thickness. Normal rumning voltage was 1450 volts, resulting in a gas gain of about 780 .

The assembled calorimeter, see Fig. 2, had three independently read out sections. The first two sections were sampled every .68 radiation length, while the third section was sampled every 1.19 radiation length. Three high voltage supplies were used, one for each section.

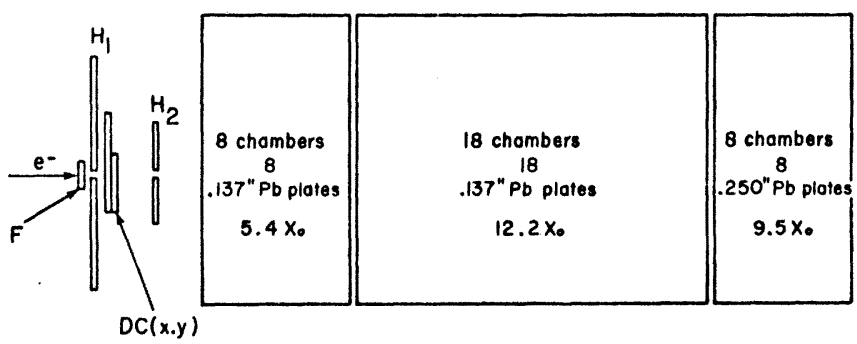

Fig. 2. Calorimeter segmentation showing beam counters and drift chambers. Each chamber represented 0.054 radiation length ( $3 / 16$ in. aluminum).

The chambers were connected to a common intake gas manifold through individual flowmeters. All chambers were carefully leak checked, and their outputs connected to a common exhaust manifold. After a period of initial flushing, the flow rate was reduced to $10 \mathrm{cc} /$ $\min$. for each chamber. No adverse effects of this low flow rate were noted.

\section{Readout Electronics}

The wires and strips were grouped two-by-two in each chamber and daisy-chained to all corresponding wires or strips in a given section along the beam direction. This gave an effective cell width of $1.2 \mathrm{~cm}$ for both wires and strips.

Fig. 3 shows the electronics connection to the chambers. The $0.1 \mu \mathrm{F}$ capacitor at the integrater input prevented any possible high voltage leakage through the $200 \mathrm{pF}$ blocking capacitor and surface leakage around the cable ends from affecting the wire amplifiers. The $50 \mathrm{ohm}$ resistor damped oscillations on the coaxial cable and improved the system noise.

The integrator, sample and hold, and differential amplifier were part of a single multiplexed ADC system. Before a trigger, both the "before" and "after" switches are closed. The trigger causes the "before" switch to open, capturing the zero level of the integrator output. The integrator response to an input charge propogates down the delay line and is placed on the "after" sample and hold capacitor. The "after" sample and hold switch is then opened and the differential amplifier takes the appropriate difference between the "before" and "after" levels so as to provide a positive output to the $A D C$.

Because of limitations in the number of available channels, section 1 had $16 \times 16$ instrumented wire and strip pairs, or an instrumented area of about $19 \times 19$ $\mathrm{cm}^{2}$. Sections 2 and 3 had $23 \times 23$ channels, or an area 


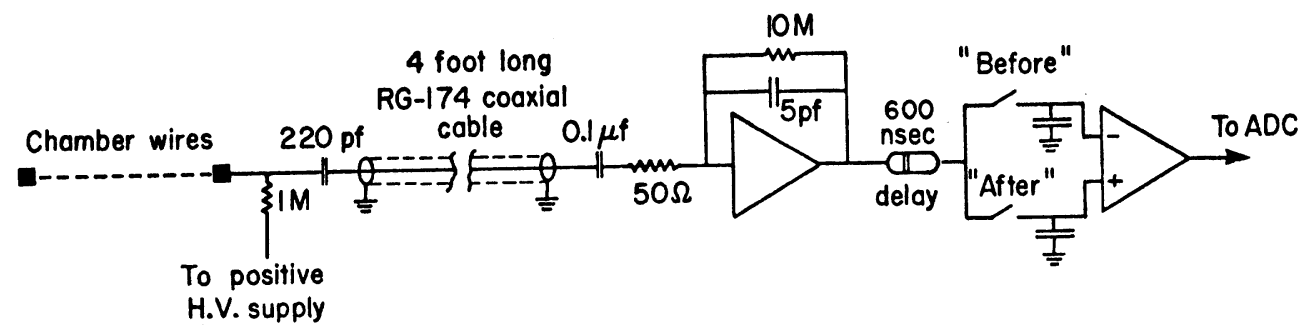

NEGATIVE SIGNAL (ANODE) AMPLIFIER CIRCUIT

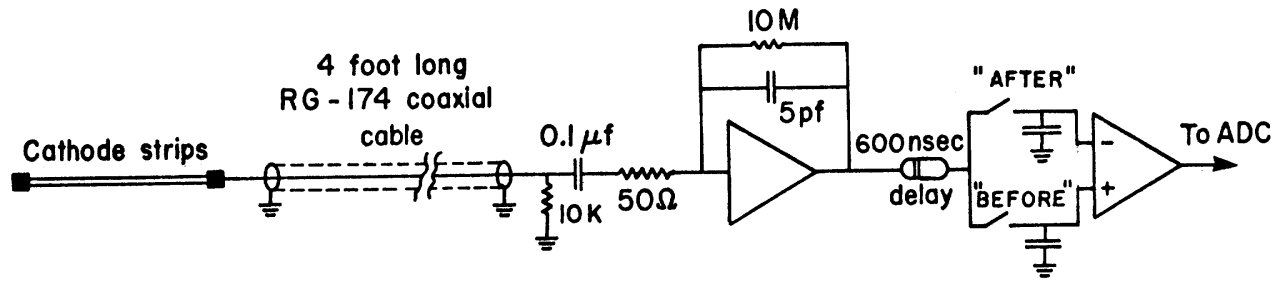

POSITIVE SIGNAL (CATHODE) AMPLIFIER CIRCUIT

Fig. 3. Readout electronics.

of $27.6 \times 27.6 \mathrm{~cm}^{2}$. Incident particles were usually kept near the center of the instrumented area to minimize energy leakage out of the wings.

The data were collected and written on magnetic tape for offline analysis. Online analysis and diagnostics were implemented using the MULTI software package distributed by the Fermilab Computing Department.

\section{Calibration}

Gas gain was monitored in one chamber of each section using the $22 \mathrm{KeV}$ line of a Cd ${ }^{109}$ source with special high gain amplifiers. A typical source peak is shown in Fig. 4. It was possible to determine the source peak position to within 18 . Source triggers were admitted during the accelerator's interspill period so that we could monitor gas gain changes during a data run.

Electronics gain was initially measured using a charge injector at the amplifier inputs. The amplifiers were then connected to the chambers, and the field wires pulsed. This provided an easy way to see time dependent gain variations, and at the same time verify the integrity of the chamber to amplifier connections. We found the amplifiers to have an r.m.s. gain variation of $3 \%$. Gain changes with time were insignificant.

\section{Beamline and Trigger}

The calorimeter was installed in the M5 beamline at Fermilab. The beam consisted primarily of pions with about $2 \%$ electrons at $40 \mathrm{GeV}$. Particle identification and beam trigger were provided by a series of Cherenkov detectors and scintillator counters. See Fig. 5. tion:

An electron signal was obtained from the combina-

$\mathrm{Cl} \cdot \mathrm{C} 2 \cdot \mathrm{U} \cdot \mathrm{D} \cdot \mathrm{F} \cdot \overline{\mathrm{H}} \cdot \overline{\mathrm{H} 2}$

A pion signal was obtained from:

$\overline{\mathrm{Cl}} \cdot \overline{\mathrm{C} 2} \cdot \mathrm{U} \cdot \mathrm{D} \cdot \mathrm{F} \cdot \overline{\mathrm{H} l} \cdot \overline{\mathrm{H} 2}$
In addition, an upper level discriminator on $\mathrm{F}$ measured $\mathrm{dE} / \mathrm{dx}$ and was used to veto multiple particles that did not diverge sufficiently to hit hole counter $\mathrm{Hl}$ or $\mathrm{H} 2$.

A pile-up gate was implemented that prevented a particle from generating a trigger when preceeded by another particle within 1 microsecond. A flag was set in a CAMAC register allowing offline rejection of events when a triggering particle was followed by another particle within the sensitive period of the readout system (I microsecond).

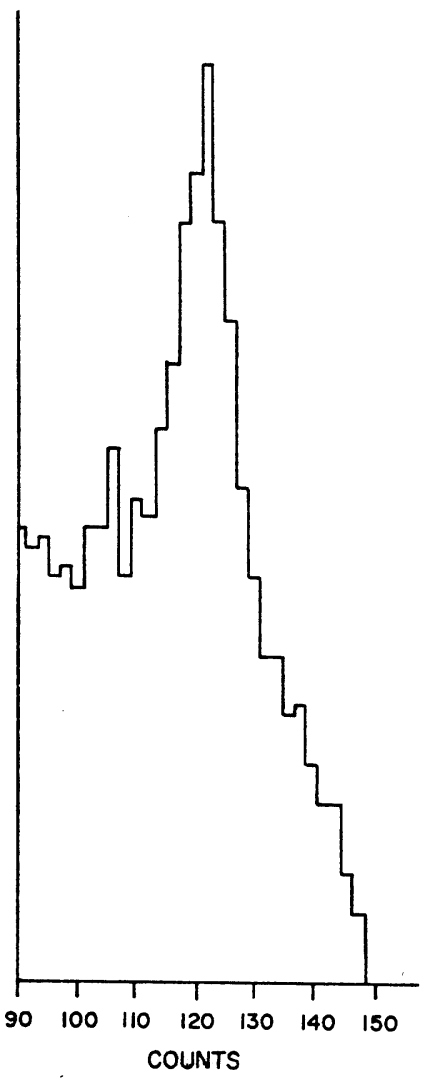

Fig. 4. Cd109 $22 \mathrm{KeV}$ line used for gas gain measurement. Chamber was operated at 1450 volts. 




Fig. 5. M5 downstream layout. Cherenkov counters $\mathrm{Cl}$ and C2 tagged electrons. Scintillator counters U (up), $D$ (down), and F (finger) indicated passage of a particle. Hole counters $\mathrm{Hl}$ and $\mathrm{H} 2$ determined the trigger beam size and indicated the presence of multiple particles from upstream secondary production. Drift chambers D. C. X and D. C. Y monitored particle position.

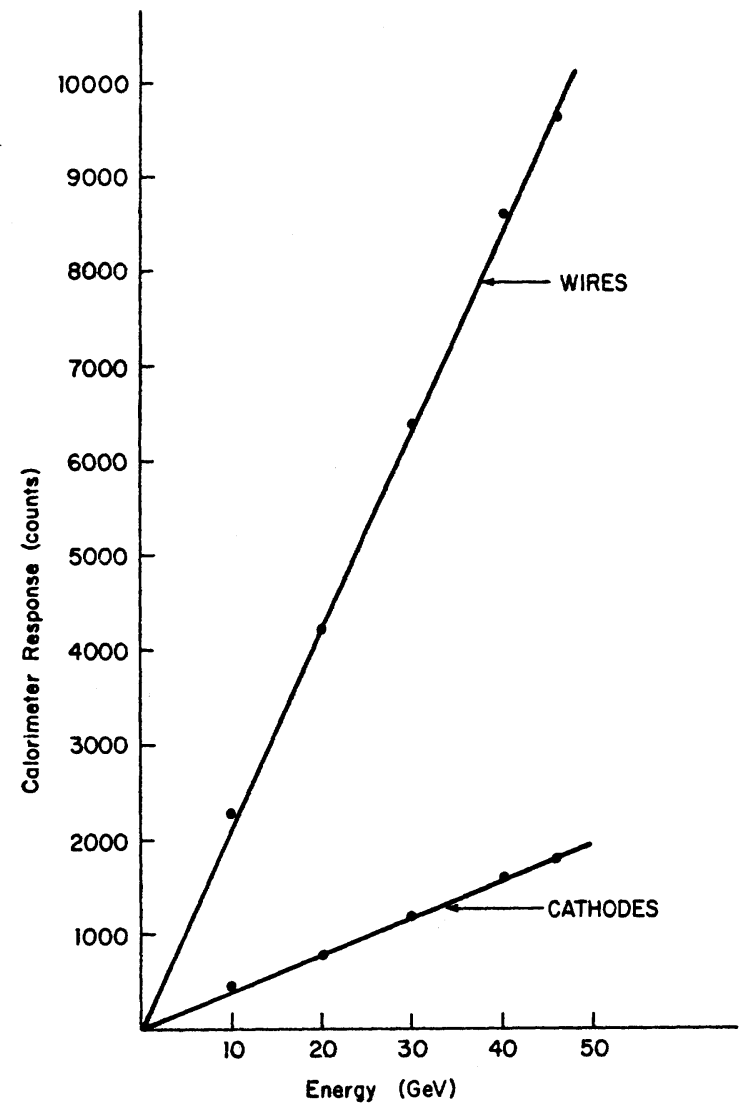

Fig. 6. Calorimeter response versus electron energy. Wire slope is 213 counts per $\mathrm{GeV}$, strip slope is 39 counts per GeV.

\section{Test Results}

Fig. 6 shows the response of the calorimeter to different energy electrons. The wire response is seen to be 5.46 times the strip response. This is due to both cell geometry, which accounts for a factor of 4, and different gain of the charge amplifiers as a function of input capacitive loading.

Energy resolution for the wires is shown in Fig. 7, and shows a $24 \% / \sqrt{E}$ dependence. Resolution is plotted both with the third section and without. The improvement seen when not using the third section shows that the noise contribution from that section is more significant than the signal content.

Cathode resolution is worse than wire resolution. about $27 \% / \sqrt{\mathrm{E}}$. This degradation is not well understood. It may be due to an unfavorable signal to noise ratio.

Fig. 8 shows a $40 \mathrm{GeV}$ electron peak with the Gaussian fit used to determine the resolution.

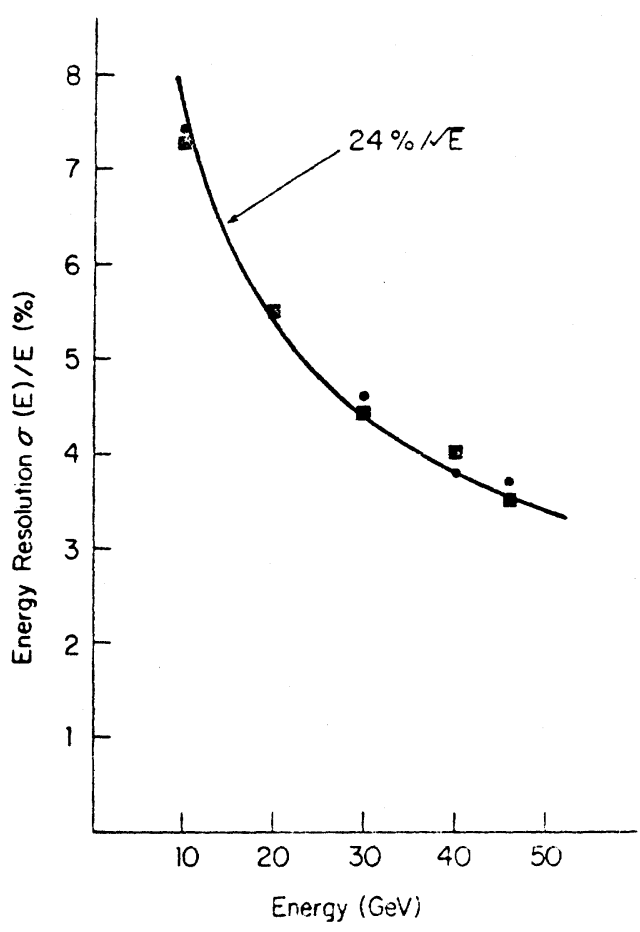

Fig. 7. Wire energy resolution. Resolution from the sum of all three sections is indicated by $\bullet$, resolution from section 1 and 2 sum only is indicated by $\mathbf{~}$.

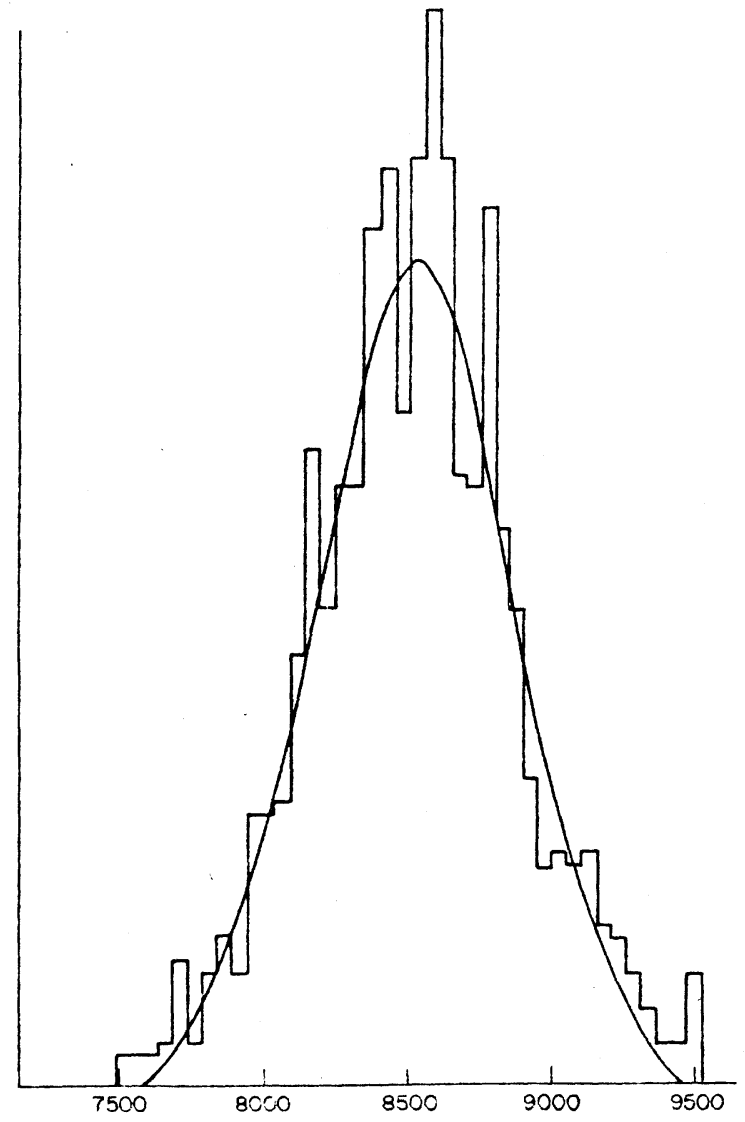

Fig. 8. $40 \mathrm{GeV}$ electron wire signal with least squares fitted Gaussian. 
Position resolution was measured at several energies for both the first and second sections. The positions of incident electrons were calculated using both the first and second moment of the lateral energy distribution. The difference between the calculated position and the particle position as measured by a drift chamber was used for position resolution determination. As seen in Fig. 9, the first section gave better resolution than the second. Also, the second moment calculation, which weights the large signal channels more than the low signal channels, gave significantly more accurate position information than the first moment calculation. First section, second moment position resolution was $1.24 \mathrm{~mm}$ at $10 \mathrm{GeV}$ and $0.84 \mathrm{~mm}$ at $46 \mathrm{GeV}$.

There was no significant difference in the position resolution of the wires versus the strips for either section or calculation method.

Studies were done to determine the effect on the total calorimeter signal and energy resolution as data were incrementally discarded from one side or both sides of the shower.

Shower position was calculated using the lateral energy distribution. The detector was made symmetric around the shower by determining the distance to the closest edge and discarding enough channels from the other edge to make both distances equal in each section. Then, since the first section had a smaller instrumented area than the others, same of the remaining channels from the second and third sections were discarded so as to make the size of the instrumented areas the same in all sections. The resulting detector measured 14 channels by 14 channels, or $16.8 \times 16.8 \mathrm{~cm}^{2}$. This configuration represents the point of zero channels cut in Figs. 10, 11, and 12 .

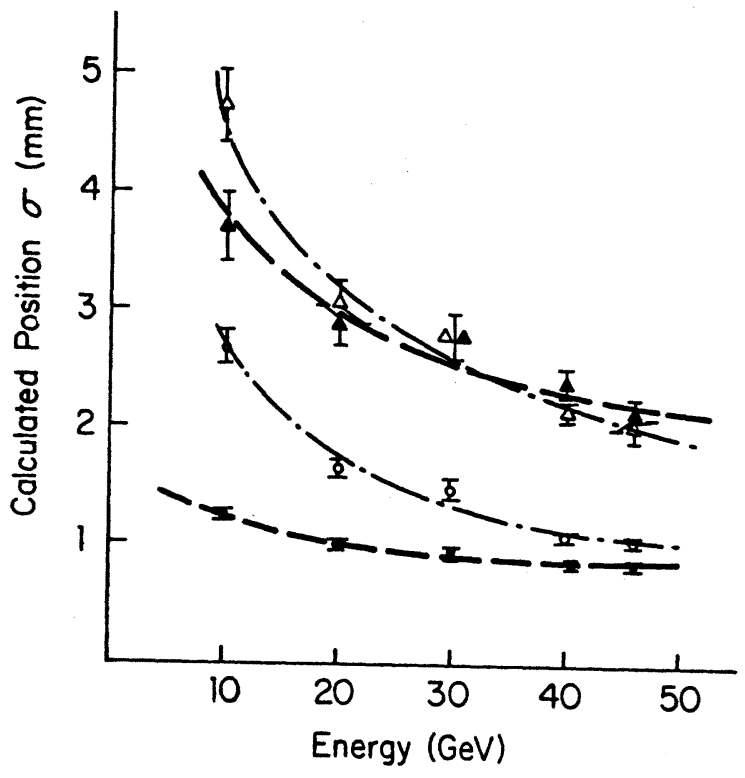

Fig. 9. Position resolution for electrons as a function of incident energy. Position was calculated using either the first or second moment of the lateral energy distribution in both sections 1 and 2 .

\section{$\Delta$ is first moment in section 2}

is second mament in section 2

$O$ is first moment in section 1

is second moment in section 1

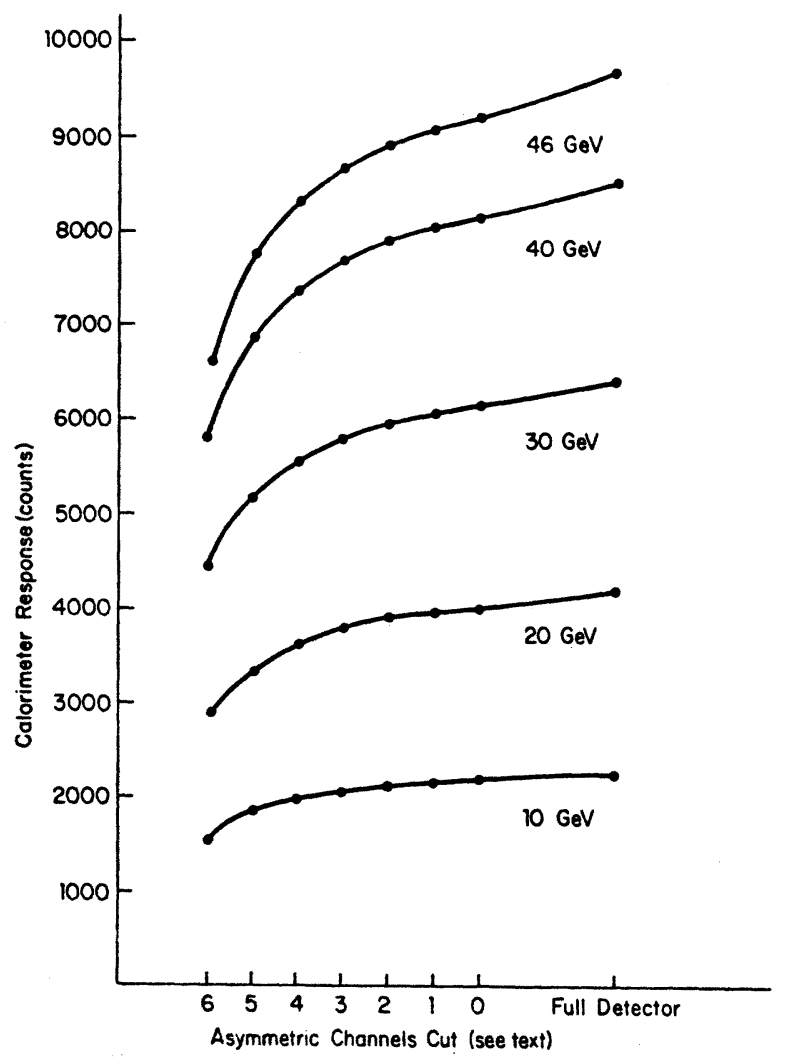

Fig. 10. Calorimeter wire signal versus number of channels cut from one side of the shower only. See text for discussion.

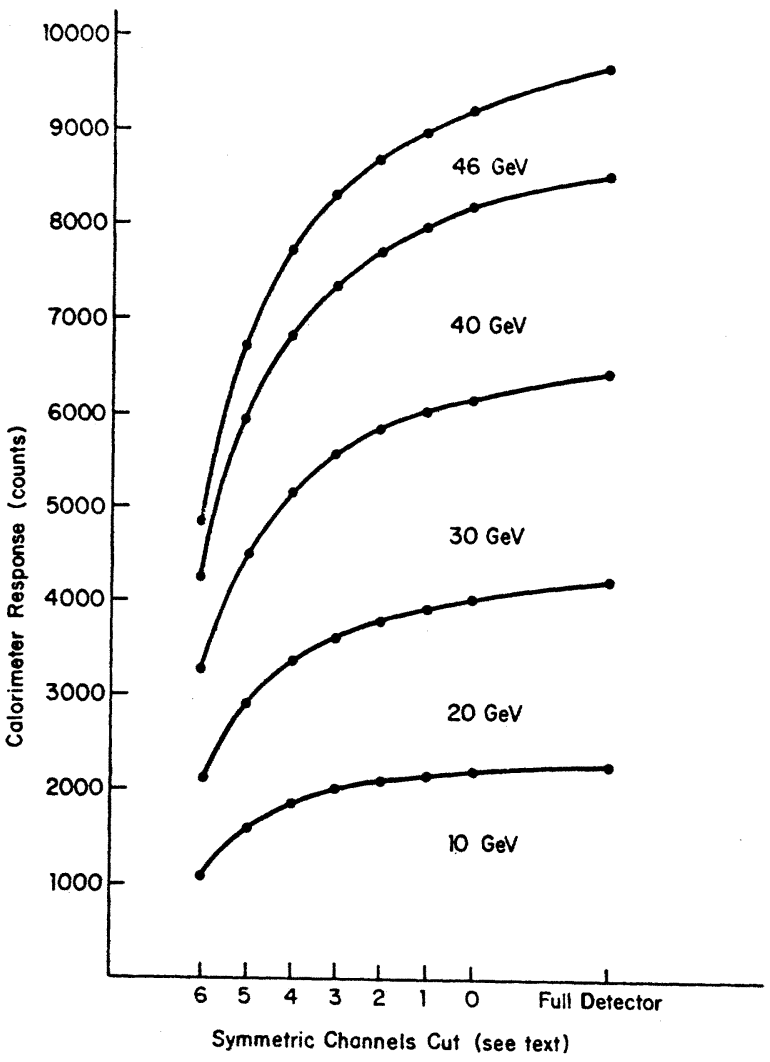

Fig. 11. Calorimeter wire signal versus number of channels cut symetrically from both shower edges. See text for discussion. 


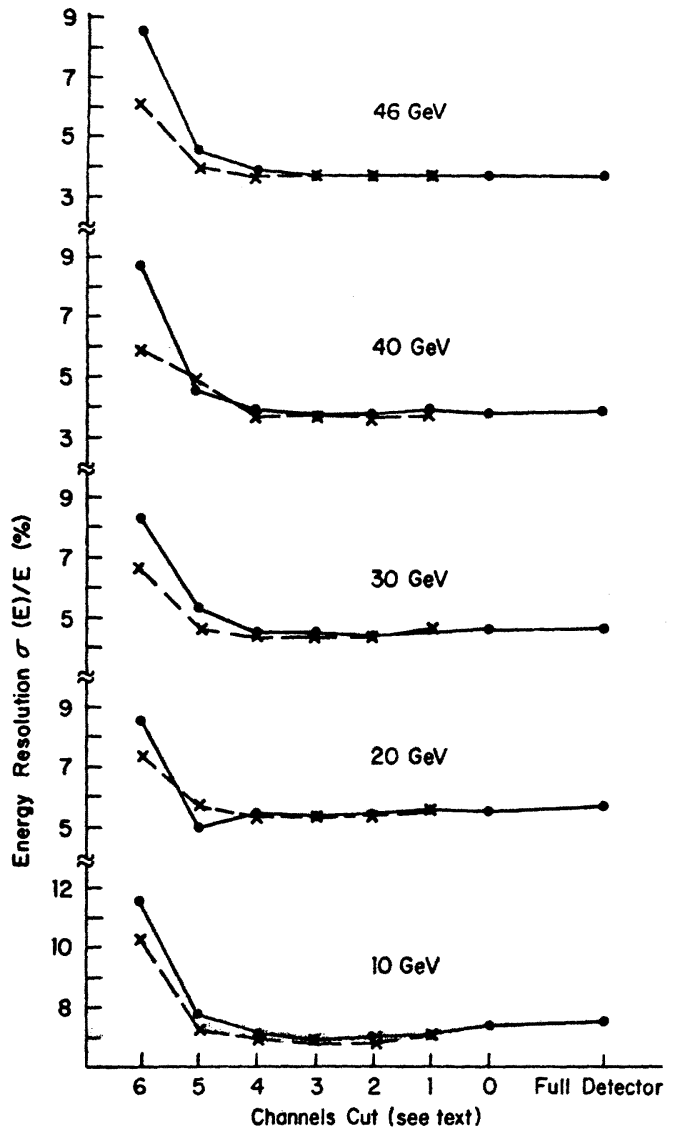

Fig. 12. Energy resolution for wires as a function of the number of channels cut fram one edge of the shower $(\bullet)$, or symmetrically from both edges $(x)$. See text for discussion.

Fig. 10 shows how the total wire signal varies when one starts with the above described configuration and removes channels from one side of the detector only. Comparison with the full detector response shows that from 3.48 at $10 \mathrm{GeV}$ to $4.7 \%$ at $46 \mathrm{GeV}$ of the signal was lost when the detector was reduced to its initial configuration. The points corresponding to six channels cut describe a detector eight channels wide $(9.6 \mathrm{~cm})$, with the shower core between the seventh and eighth cells.

Fig. 11 shows the wire signal as a function of symmetric cuts on both detector edges. The final points with six channels symetrically cut describe a detector two channels wide with the electron shower core between those two channels.

Fig. 12 shows how the energy resolution changes as a function of both symmetric and asymmetric cuts. Interestingly, the resolution shows some improvement, particularly at lower energies, as one discards the extreme shower edges. Also note that the resolution resulting from two channels alone (six channels cut symetrically) is considerably better than the resolution resulting from an asymmetric cut of six channels, where one wing is included.

Hadron rejection was measured at $40 \mathrm{GeV}$ only. A lead brick was inserted far upstream of the detector to attenuate the electron fraction prior to trigger formation. Fig. 13 shows the pion peak with a superimposed curve showing the position of the $40 \mathrm{GeV}$ electron peak.
Applying a total signal cut $7.1 \mathrm{GeV}$ wide centered on the $40 \mathrm{GeV}$ electron peak discarded about 28 of the electrons, The resulting pion rejection factor was $8.4 \times 10^{-3}$. An additional cut was made on the section 1 signal. Discarding $10 \%$ of the electrons, the two cuts together gave a rejection factor of $4 \times 10^{-4}$. Fig. 14 shows the application of those cuts to the pion signal.

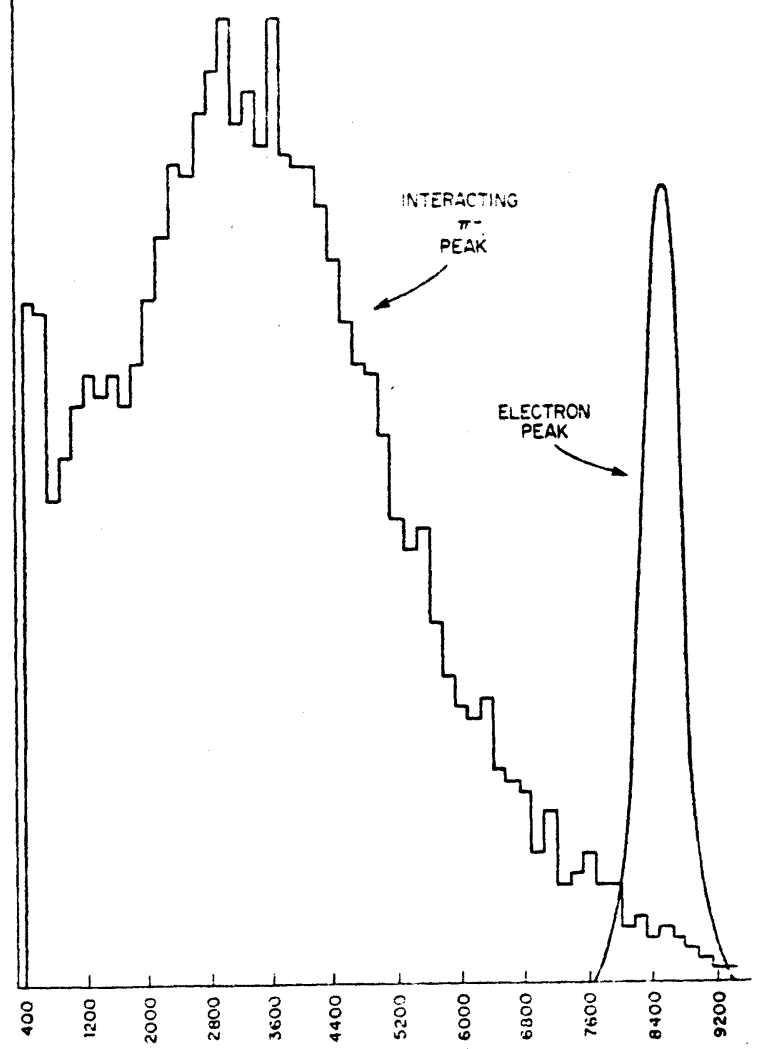

Fig. 13. $40 \mathrm{GeV}$ pion peak showing position of $40 \mathrm{GeV}$ electron peak on same scale. The minimum ionizing peak is not shown.

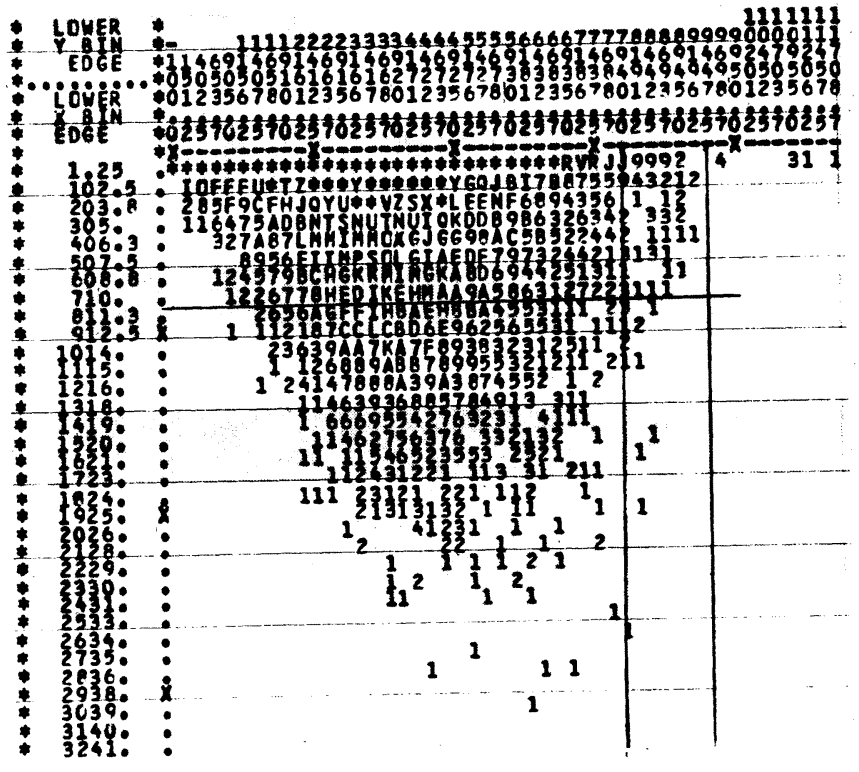

Fig. 14. Correlation plot of total calorimeter signal (horizontal axis) against first section signal (vertical axis) for $40 \mathrm{GeV}$ pions. Lines show the position of the cuts used to determine the pion rejection factor. 


\section{Acknowledgments}

We would like to thank Dr. M. Mishina, Dr. J.

Saver, W. Coleman, M. Davis, M. Hrycyk, and E. Mabeus

for their help in conducting these tests.

\section{References}

1. R. L. Anderson, W. W. Ash, D. B. Gustafson, K. Rich, D. M. Ritson, J. R. Johnson, R. Freeport, and D. E. Wiser, IEEEE NS-25 (1978) 340.

2. P. Skubic, F. Sannes, D. Potter, J. J. Mueller, R. Imlay, G. K. Chang, and D. Bechis, contributed to this conference.

3. T. F. Droege, M. C. Hibbard, C. A. Nelson, Jr., P. A. Thampson, Y. Makdisi, R. Lipton, IFEFE NS-27 (1980) 64

4. J. F. Bartlett, J. R. Biel, D. B. Curtis, R. J. Dosen, T. D. Lagerlund, D. J. Ritchie, and L. M. Taff, IFEEE NS-26 (1979) 4427.

*operated by Universities Research Association under Contract with the United States Department of Energy. 